\title{
LONGITUDINAL DENSITY MODULATION OF UNSTABLE BUNCHES EMITTING COHERENT IR*
}

\author{
B. Podobedov, G.L. Carr, S.L. Kramer and J.B. Murphy \\ National Synchrotron Light Source, Brookhaven National Laboratory, Upton, NY 11973
}

\begin{abstract}
Observations of coherent infrared (IR) light emissions from $\sim 30 \mathrm{~cm}$ long electron bunches at the NSLS VUV ring were reported in [1]. The emissions occurred above some current threshold and had a maximum spectral content near $42 \mathrm{GHz}$, leading to speculations that they were due to a longitudinal instability creating a $\sim 7 \mathrm{~mm}$ modulation of the bunch density. To check this experimentally we performed streak camera measurements recording a large number of beam profiles taken both during and between the coherent emission bursts. In the course of our studies we have directly proven that the longitudinal beam density indeed experiences a significant modulation when coherent emission bursts occur.
\end{abstract}

\section{MOTIVATION AND SCOPE}

The NSLS VUV ring is a dedicated synchrotron radiation source operating at $800 \mathrm{MeV}$. Built in the early 1980s, without thorough impedance budget calculations, it nevertheless operates at a fairly high single bunch intensity. During regular operations $1 \mathrm{~A}$ of current is injected into 7 out of 9 buckets of $52.9 \mathrm{MHz}$ RF, while for studies up to 400 $\mathrm{mA}$ could be stored in a single bunch. Such a performance deserves better understanding that should come from studies of high current single bunch dynamics in the VUV ring.

One of many interesting collective phenomena in the VUV ring is the recently reported [1] effect of coherent IR light emissions. It was first observed using an interferometer with a bolometric detector installed at one of the IR beamlines. When the VUV ring was operated above a single bunch current threshold, this system (reportedly sensitive in the 30-3000 GHz frequency range) recorded quasiperiodic bursts of coherent radiation. At the observed peak in the spectral contents of emissions at $42 \mathrm{GHz}$ the coherent power exceeded the incoherent by more than a factor of $10^{4}$. Some aspects of the threshold dependence on current were consistent with the microwave instability, and the coherent emissions were speculated to be due to the instability induced longitudinal beam density modulation.

To check this conjecture experimentally we performed detailed bunch profile measurements looking at the visible synchrotron light from a bending magnet with a streak camera. This device directly measures the bunch shape in the time domain, and, in contrast to the interferometer system, it has virtually no low frequency cutoff and does not require any normalization of data.

\footnotetext{
${ }^{*}$ Work supported by US DOE under contract DE-AC02-98CH10886
}

\section{EXPERIMENTAL SETUP}

\subsection{Coherent Burst Signal}

We used two different approaches to identify the time intervals when coherent bursts occur. First, we looked at a button BPM ( $4 \mathrm{GHz}$ bandwidth) pickup signal that was detected with a circuit described in [2]. Above the instability threshold we did see this signal to acquire transitions correlating with the coherent emission bursts.

In the other, more direct method, we looked at the power of microwave signals coming from the IR port on the same beamline the original interferometer experiment was made. To receive the radiation coming from the beam port we used either a microwave horn operating in $33-50 \mathrm{GHz}$ band or a $1 / 4$ wave antennae (probe) sensitive in $\sim 3-15 \mathrm{GHz}$ range. Each one was followed by a suitable microwave detector with a video bandwidth in excess of $10 \mathrm{MHz}$.

Oscilloscope traces of the detected signal received by the horn at different beam currents are plotted in Fig. 1. It shows 100 to $200 \mu$ s long spikes, pointing down due to a negative detector used, which decline in numbers and amplitude as beam current decays until they completely disappear below the threshold (bottom trace). Note that in this figure and in Figs. 2 and 3 to follow, the vertical scale is the same for all traces but the origin is shifted to avoid overlap.

Setting up this signal we found that the frequency content of the microwave emissions from the dipole is more complex than a single $42 \mathrm{GHz}$ line, and that there are significant emissions at lower frequencies. In addition, we observed the time structure of the microwave bursts to vary with frequency. This is illustrated in Fig. 2, where we plot simultaneously taken traces of detected microwave signals

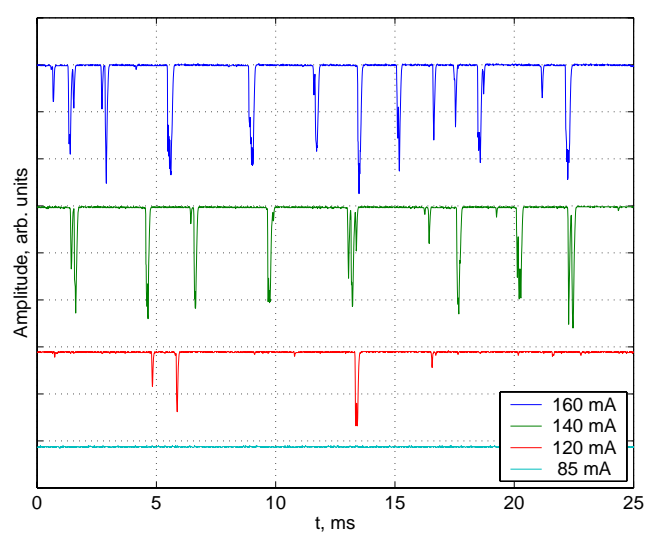

Figure 1: Detected microwave signals. 

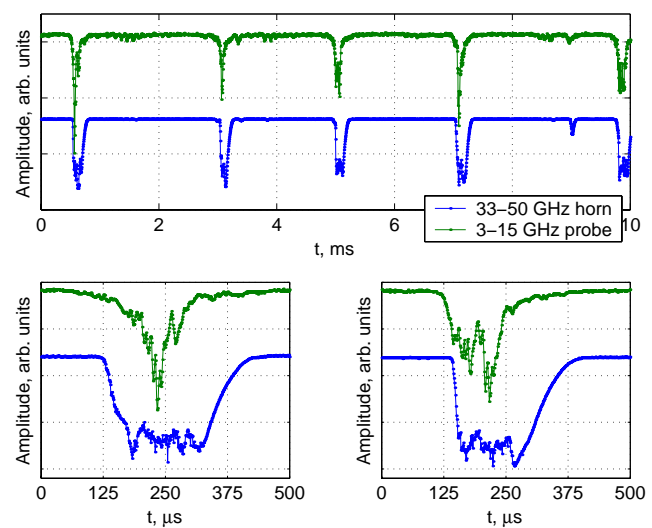

Figure 2: Detected signals at two different MW bands.

received by the probe and the horn. It shows (in agreement with large number of traces recorded) that on a ms time scale the bursts generally coincide (Fig. 2, top), however, depending on the frequency, the time structure of an individual burst may be different (two bottom sub-plots).

This demonstrated that coherent emission bursts have varying spectral content. Therefore we used a signal from the horn to derive the streak camera trigger when searching for beam density modulation in the vicinity of $42 \mathrm{GHz}$, and the probe detector was used for lower frequencies.

More on VUV ring coherent emissions is reported in [3].

\subsection{Streak Camera}

We used a Hamamatsu model C5680 streak camera for this experiment. Visible synchrotron light from a dipole magnet was brought to the camera by a series of mirrors. The camera was operated in a single sweep mode and, to increase the sensitivity, we used cylindrical lens to spread the light along the photocathode. To get the longitudinal beam profile the final image was integrated in the CCD. An interference filter with $500 \pm 40 \mathrm{~nm}$ passband was used to alleviate possible chromatic effects. The $106 \mathrm{MHz}$ synchroscan unit (with internal frequency doubler) was connected to a pickup signal from the ring main RF cavity.

Generally, to achieve the optimum signal/noise ratio in a synchroscan camera, one has to integrate over as many incoming light pulses as possible, provided that the bunch distribution remains frozen. Assuming the fastest change in the latter is due to the phase space rotation, to detect a density modulation of size $\delta$, the integration time $\Delta T$ must be much shorter than $f_{s}^{-1} \delta / \sigma$, where $f_{s}$ is the synchrotron frequency and $\sigma$ is the bunch length. As we found experimentally, a few $\mu$ s was an optimum value for our setup, and the data described later in this paper was taken at $\Delta T=3 \mu \mathrm{s}$ (18 passages of electron bunch).

To select a particular sequence of beam turns we controlled the streak camera gain by supplying C5680 with an external trigger. It was derived from the detected burst signal using a Logic Trigger feature of Tektronix TDS 620 oscilloscope. This essentially allowed us to set a threshold on the burst amplitude, giving a logic pulse at the threshold crossings. The beam profiles within the bursts were taken with the threshold set to $\sim 80 \%$ of the maximum burst amplitude at a given current. Similarly, to record between the bursts profiles, we set the threshold to $<5 \%$ of the burst amplitude and inverted the logic.

\section{EXPERIMENT AND RESULTS}

Similar to [1] our experiment was done with a single bunch at a ring energy of $737 \mathrm{MeV}$. The fourth harmonic $\mathrm{RF}$ cavity used in regular operations to increase the lifetime was normally detuned and set to low power. Relevant ring parameters include synchrotron frequency $f_{s}=12 \mathrm{kHz}$, low current rms energy spread $\delta E / E=5 \times 10^{-4}$, momentum compaction $\alpha=0.0235$, and radiation damping time $\tau_{d}=$ $10 \mathrm{~ms}$. A more detailed list may be found in [4]. In this configuration the instability threshold was about $100 \mathrm{~mA}$.

During our studies a single bunch current of up to 300 $\mathrm{mA}$ was injected into the ring. As it decayed we recorded streak camera profiles interleaving sets of "bursting" and "non-bursting" beam by resetting the TDS 620 logic trigger. Each set typically contained a few hundred profiles recorded fast enough for current to decay less than $5 \%$.

While taking data we noticed a difference between the profiles from non-bursting and bursting sets. While the former didn't show any special features, many of the bursting profiles had some modulation. Surprisingly, it was mostly at 6-7 $\mathrm{GHz}$ rather than at $42 \mathrm{GHz}$ prompting us to switch to the probe-based trigger. After that we observed some modulation in virtually every profile in the bursting set.

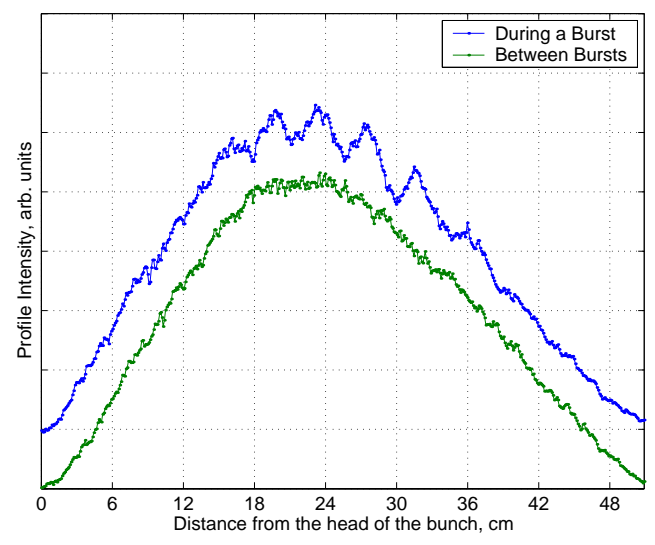

Figure 3: Typical bursting and non-bursting profiles.

Fig. 3 shows a typical profile from a bursting set (top trace) and a non-bursting one. Both sets were taken at about $250 \mathrm{~mA}$.

While most of the non-bursting profiles looked quite similar to each other, the bursting ones did not. The number, location and to some extent the size of the modulation lobes did vary between the profiles. This is why to quantitatively describe the average difference between the bursting 


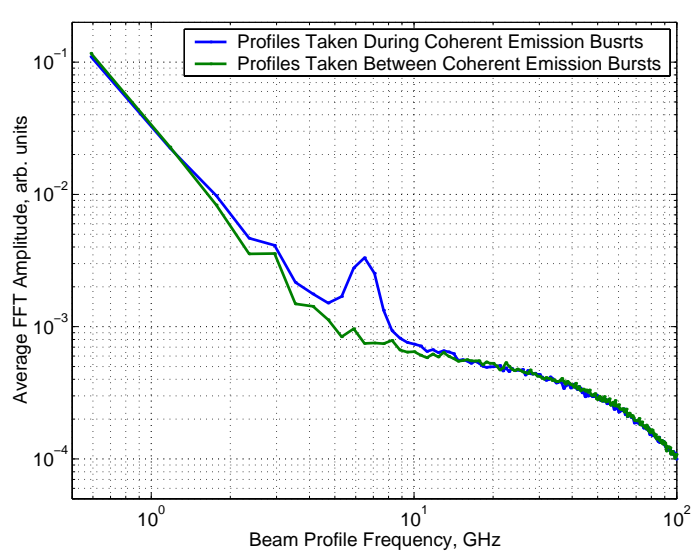

Figure 4: Average FFT amplitudes of beam profiles.

and non-bursting data we switched to the frequency domain. We applied an FFT to each individual profile and then averaged the FFT amplitude over each 300-profile set. The resulting averages are plotted in Fig. 4. It shows that in the $\sim 1-15 \mathrm{GHz}$ frequency range the spectral contents of the bursting profiles exceeds that of the non-bursting ones. At the peak at $6.5 \mathrm{GHz}$ the average FFT amplitude of the bursting set is higher by more than a factor of 4 .

Both traces in Fig. 4 exhibit a common trend, that is a rapid decline at low frequency, consistent with $\sim 1$ ns FWHM average bunch length, a "plateau" region in the middle, and finally a sharp decline, due to CCD digitization. The amplitude at the plateau is mostly due to the residual noise in individual streak camera profiles, such as seen in Fig. 3. Our low current measurements have shown this noise to scale as a square root of the incoming light intensity, which is consistent with photoelectron shot noise in the camera. To reduce this noise we could either increase the light intensity (but not easily since we were using the maximum available intensity from this beamline), or integrate for a longer time. Using $\Delta T>10 \mu$ s reduced the noise in the plateau region, but did not reveal any new spectral features. Instead, we observed a decrease in the $6.5 \mathrm{GHz}$ peak, presumably due to phase variations of this signal relative to the RF trigger.

The observed $6.5 \mathrm{GHz}$ modulation frequency peak coincides with the largest peak in the longitudinal impedance calculated for RF shield for the VUV ring vacuum chamber bellows [5]. It is not surprising that, when the threshold for the microwave instability is exceeded, the largest density modulation occurs at this frequency providing the bunch is long enough to support it.

To substantiate this further we repeated our measurements in other ring configurations allowing for much shorter bunch length. The latter can be achieved in the VUV ring by lowering $\alpha$ or running the harmonic RF in the bunch compressor mode. For example in one particular configuration (that used both) we stored $15 \mathrm{~mA}$ of current with $f_{s} \sim 6 \mathrm{kHz}$ and the average bunch length a factor of 4 lower compared to the previous data. For this experiment the trigger was derived from the horn. Performing similar data analysis as was done for Fig. 4 we observed the $6.5 \mathrm{GHz}$ peak to disappear and the modulation frequency to shift into $\sim 8$-to-mid-40 GHz range.

Similarly, in other configurations shortening the bunch caused the frequency of beam density modulation during coherent emissions to move up. This is consistent with the longitudinal instability developing at higher frequencies as the bunch samples higher frequency impedance.

\section{SUMMARY AND OUTLOOK}

We have directly shown that the electron bunch in the NSLS VUV ring contains a significant longitudinal density modulation when a burst of millimeter wave coherent synchrotron radiation is emitted. For conditions of no bunch compression and the ring's normal lattice, the spectral content extends to $15 \mathrm{GHz}$, with the largest component at 6.5 $\mathrm{GHz}$, corresponding to the highest peak in the RF bellow shield longitudinal impedance. When the ring is operated with shorter bunches the range of modulation frequencies extends above $40 \mathrm{GHz}$, but with no distinct peak. Our measurements are consistent with the hypothesis that a microwave instability, induced by a broad band impedance of the vacuum chamber, creates a density ripple on the beam causing it to radiate coherently in this frequency range.

Our measurements do not show a $7 \mathrm{~mm}$ density modulation consistent with the $42 \mathrm{GHz}$ emission frequency reported in [1]. Although we cannot exclude measurement uncertainties in [1] as the cause, a more likely explanation is that the streak camera is not sensitive enough for detecting a small density modulation at this frequency. If our preliminary estimate of $\sim 30 \mathrm{GHz}$ cutoff for the coherent synchrotron radiation [6] for the IR beamline is correct, the beam density ripple at $42 \mathrm{GHz}$ must be extremely low in proportion to the $6.5 \mathrm{GHz}$ one, since the observed emission power at these frequencies has the same order of magnitude.

We plan further experiments with modified input apertures for collecting more light and improved streak camera sensitivity to higher frequency density modulations.

\section{ACKNOWLEDGEMENTS}

We wish to thank Sam Krinsky and Nathan Towne of NSLS for stimulating discussions and William Cieslik of Hamamatsu for technical assistance. This work was supported by the DOE under contract DE-AC02-98CH10886.

\section{REFERENCES}

[1] G.L. Carr et. al., NIM A, 463 (2001), 387.

[2] B. Podobedov and R.H. Siemann, Proc. 1997 PAC, 1629.

[3] G.L. Carr and S.L. Kramer, this conference.

[4] http://nslsweb.nsls.bnl.gov/operations/.

[5] S.L. Kramer and A. Novokhatski, Private Communication.

[6] R.L. Warnock and P. Morton, Part. Accel., 25 (1990), 113. 